Original Research

\title{
Operation of AC Microgrids with PV Panels' Output Power Curtailment for Minimizing the Use of Energy Storage
}

Daming Zhang *

University of New South Wales, Sydney, Australia; E-Mail: daming.zhang@unsw.edu.au

* Correspondence: Daming Zhang; E-Mail: daming.zhang@unsw.edu.au

Academic Editor: Rodolfo Dufo-López

Journal of Energy and Power Technology

2022, volume 4, issue 1

doi:10.21926/jept.2201008
Received: January 21, 2022

Accepted: February 24, 2022

Published: March 07, 2022

\begin{abstract}
In this paper, a method for operating AC microgrids in both off-grid or standalone and gridconnected modes is proposed to minimize the use of energy storage by increasing the number of PV panels and curtailing their output power. When the intensity of daylight decreases, power is imported from the main grid through an AC/DC/AC converter or solid-state transformers in parallel to compensate for the shortage. By using a three-phase transformer in $\Delta-Y$ connection with $Y$ 's neutral connected to its local grounding system, the effect of unbalanced loads on the AC microgrid is minimized. The AC microgrid is operated at a constant frequency with necessary reactive power compensation, and power qualities of both voltage and frequency can be ensured.
\end{abstract}

\section{Keywords}

AC microgrid; constant frequency; curtailment; energy storage; maximum power point tracking; PV panel

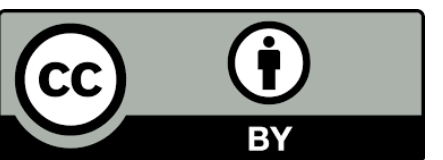

(C) 2022 by the author. This is an open access article distributed under the conditions of the Creative Commons by Attribution License, which permits unrestricted use, distribution, and reproduction in any medium or format, provided the original work is correctly cited. 


\section{Introduction}

As fossil fuels will be depleted eventually, the endeavor for harnessing renewable energy has intensified in recent years [1-5]. Several factors need to be addressed for increased utilization of renewable energy, such as environmental-friendliness, cost-effectiveness, sustainability, and persuading interest groups. To cope with the intermittency of renewable energy generation, different types of energy storage are being adopted. Battery storage is one of them and is very compact, but it involves the use of rare earth metals. Furthermore, batteries produce environmentpolluting side products. In comparison, PV panels pose less threat or perils.

Frequent charging and discharging of batteries shorten their lifespan. In addition, the lifespan of PV panels is several times greater than that of batteries. Moreover, after use for several years, the efficiency of batteries deteriorates quickly. In view of this, the use of PV panels is more economical.

To reduce the impact of AC microgrids on the stability of large grids, planned power transfer is preferable instead of dynamic power exchange. To minimize the effect of intermittency of solar power generation on large grids and reduce the use of battery storage, suitable operation methods need to be developed. One potential solution is AC microgrids, where the PV panels are controlled by curtailing the output power [6]. On days with bright daylight, $A C$ microgrids can be run without using energy storage and in autonomous mode. On days with less sunshine, some power needs to be imported from the main grid to compensate for the shortage. On overcast days, all the power must be imported from the main grid. A large energy storage farm with solar and/or wind power generation capability and connected with the AC microgrid through transmission lines and stepup/step-down transformers can be built; the required power can be transferred from such an energy storage farm. Furthermore, multiple solid-state transformers (SSTs) with medium-frequency transformers in parallel can be adopted to interface the energy storage farm with the transmission line through a 50-Hz step-up transformer.

Researchers have adopted frequency against real power and voltage against reactive power droop control with both frequency and voltage to be regulated; this makes system control complicated [1-5]. The main drawback of droop control is that it is difficult to cope with sudden and random load changes. For predictable load changes, droop control coefficients can be varied to set the system frequency within the deviation limit. However, for unpredictable load changes, techniques are yet to be developed to overcome such an obstacle. To simplify the control, constantfrequency control methods proposed in previous studies [6-9] can be adopted wherein the gridforming generator produces a reference voltage with a constant frequency, and the grid-supporting and grid-feeding generators take the voltages at their respective point-of-coupling (PCC) as reference. Therefore, the whole system operates at a constant frequency. To balance reactive power demand and generation, the grid-supporting and grid-feeding generators output around $50 \%$ of their real power output. The remaining reactive power demands are provided by the grid-forming generator and reactive power compensators. In this paper, the microgrid is operated at a constant frequency as described in previous studies [6-9], and the output power of PV panels installed in the microgrid is curtailed to reduce battery usage.

The remaining paper is organized as follows. In Section II, the configuration of the autonomous microgrid under study and its control are described. In Section III, the results and discussions are presented. Finally, conclusions are presented in Section IV. 


\section{Configuration of Grid-connected AC Microgrid and Its Control}

\subsection{Configuration of the Grid-connected AC Microgrid under Study}

Figure 1 shows the configuration of the grid-connected AC microgrid under study; it includes one grid-forming generator DG1 powered by PV panels and accompanied by a reactive power compensator DG1A. The grid-supporting generator DG2 and the grid-feeding generator DG3 are powered by PV panels. In practice, wind energy can also be employed as the source. In this study, DG1 and DG2, which are powered by solar energy by using PV panels, are operated via power curtailment. Their output power is produced by following the power reference $[6,7]$ :

$$
P_{\text {ref }}=P_{\text {ref } 0}+K_{v 0} \times\left(K_{v} \times V_{\text {ref }}-V_{m}\right) \text {, }
$$

where $V_{m}$ is the RMS value of the AC voltage at the inverter output and $V_{\text {ref }}$ is the reference RMS value.

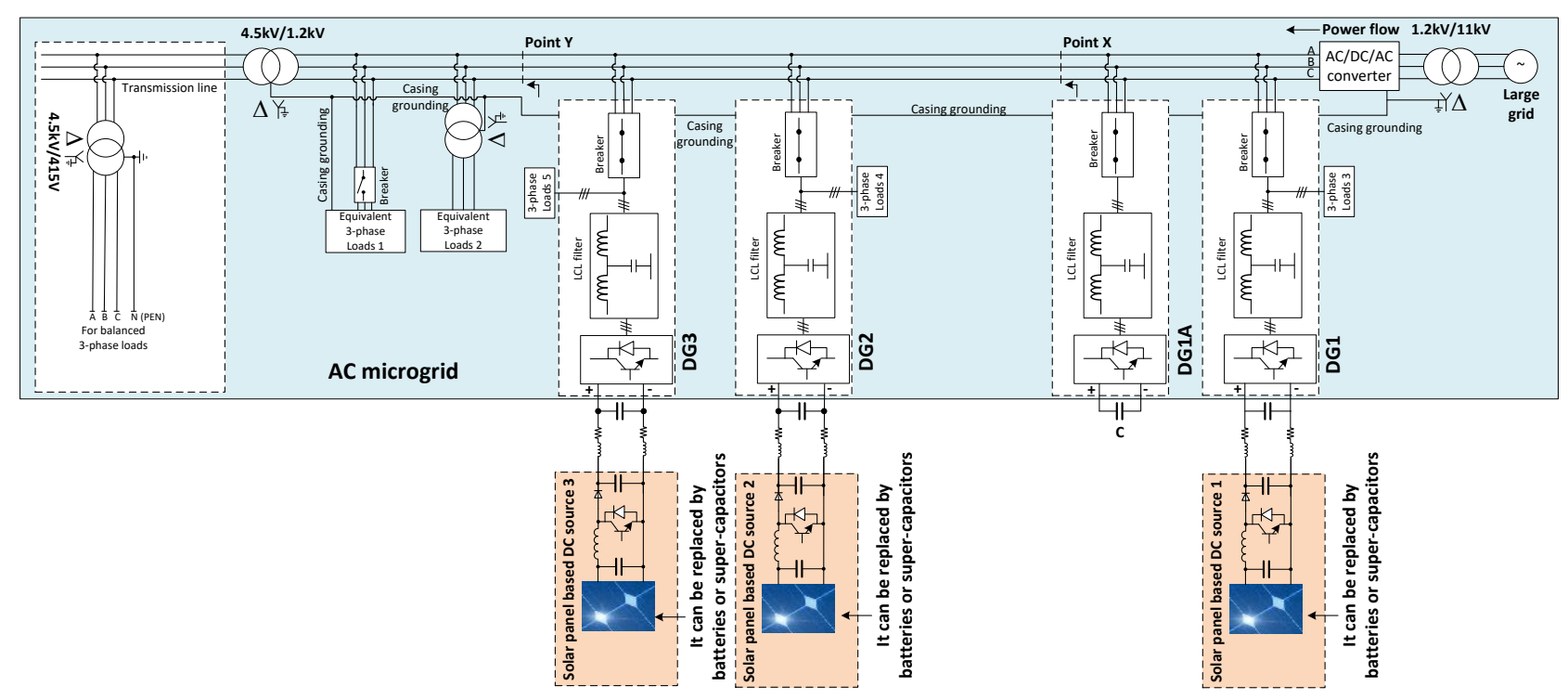

Figure 1 Configuration of the grid-connected AC microgrid under study.

The power reference $P_{\text {ref }}$ is capped at $95 \%$ of the maximum available PV power. More details are presented in the subsequent paragraphs.

DG3 is operated using the maximum power point tracking method described in a previous study [6].

The grid-supporting and grid-feeding generators output around 50\% of their respective real power output and can thus fulfill only a part of the reactive power demand; This results in a shortage of reactive power generation demanded by the loads. To achieve reactive power balance, a reactive power compensator DG1A is installed that fulfills the purpose of directional reactive power compensation; it provides the reactive power seen from location $X$ toward the left-hand side in Figure 1. By doing so, the grid-forming generator only needs to output real power, and its reactive power output fluctuates around zero.

One side of an $11 \mathrm{kV} / 1.2 \mathrm{kV}$ step-down transformer is connected with the 11-kV main grid. An $A C / D C / A C$ converter with an LCCL filter is used to control the power transfer from the main grid to 
the AC microgrid. Such an AC/DC/AC converter can be replaced by multiple SSTs in parallel. Although LCCL-based inverters can transfer more power, it is difficult to manufacture large current-carrying AC capacitors. Therefore, SSTs in parallel with medium-frequency transformer isolation are a better choice.

A step-up transformer in $Y-\Delta$ connection is used at point $Y$ when the loads are far away from the inverter-based generators. When a step-up transformer in $\Delta-Y$ connection is used at the inverter side, step-down transformers need to be used at the load side.

Figure 1 shows the three-phase transformer in $\Delta-Y$ connection with $Y^{\prime} s$ neutral connected to its local grounding system at the load side. Such a transformer can reduce the effect of unbalanced loads on the operation of inverter-based generators. Negative-sequence current compensators can be used to make the system more balanced for the inverters, even in the case of more severe unbalanced loads.

The design of the controller (Figure 2) for the PV panel is based on the transfer function (Eqs. (2) and (3)). The circuits of the controller are shown in Figure 3. The parameters used for the DC/DC converter are listed in Table 1.

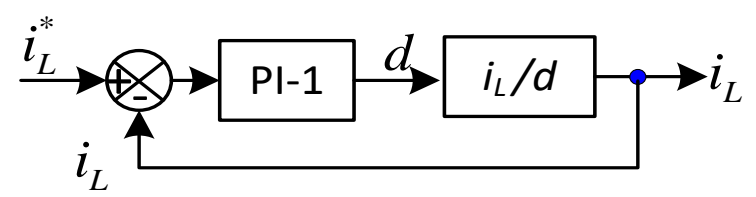

Figure 2 MPPT control: I control.

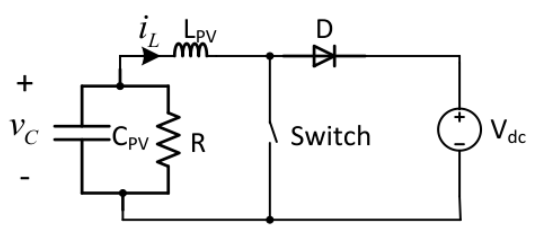

(a)

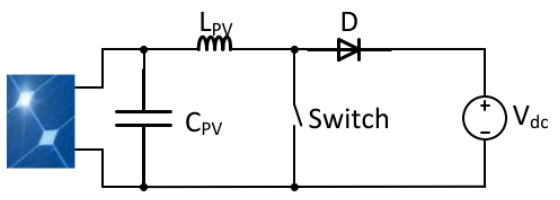

(b)

Figure 3 (a) Modified boost converter; (b) Boost converter with solar panel.

Table 1 Parameters for PV panel part.

\begin{tabular}{ll}
\hline Parameters & Values \\
\hline LPV & $0.8 \mathrm{mH}$ \\
CPV $_{P V}$ & $0.5 \mathrm{mF}$ \\
fswitching & $10 \mathrm{kHz}$ \\
fsampling & $150 \mathrm{kHz}$ \\
\hline
\end{tabular}




$$
\begin{gathered}
\left.\frac{\widehat{l_{L}}(s)}{\hat{d}(s)}\right|_{\hat{v}_{d c(s)=0}}=\frac{s+\frac{1}{R C_{P V}}}{s^{2}+\frac{1}{R C_{P V}} s+\frac{1}{L_{P V} C_{P V}}} \cdot \frac{\bar{V}_{d c}}{L_{P V}} \\
\left.\frac{\widehat{v_{c}}(s)}{\hat{d}(s)}\right|_{\hat{v}_{d c(s)=0}}=\frac{1}{s^{2}+\frac{1}{R C_{P V}} s+\frac{1}{L_{P V} C_{P V}}} \cdot\left(\frac{-\bar{V}_{d c}}{L_{P V} C_{P V}}\right)
\end{gathered}
$$

\subsection{Configuration of the Grid-connected AC Microgrid under Study}

In this paper, the control flow (Figure 4) is adopted for controlling the inverters [9-11]. A bandpass filter is used to filter out the DC and high-frequency components in the shunt-capacitor currents. Furthermore, a low-pass filter is added to remove noise from the grid-side current.

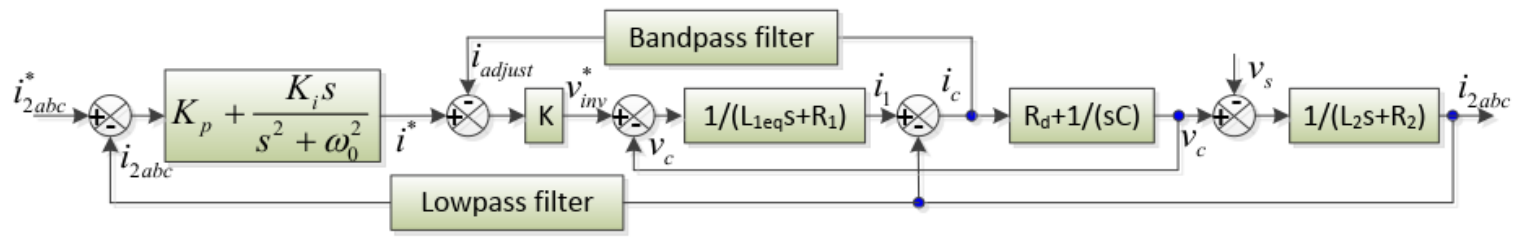

(a)

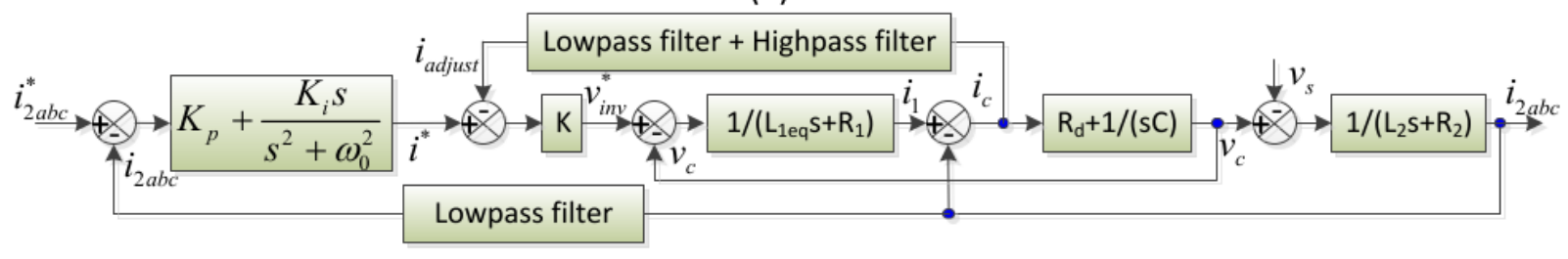

(b)

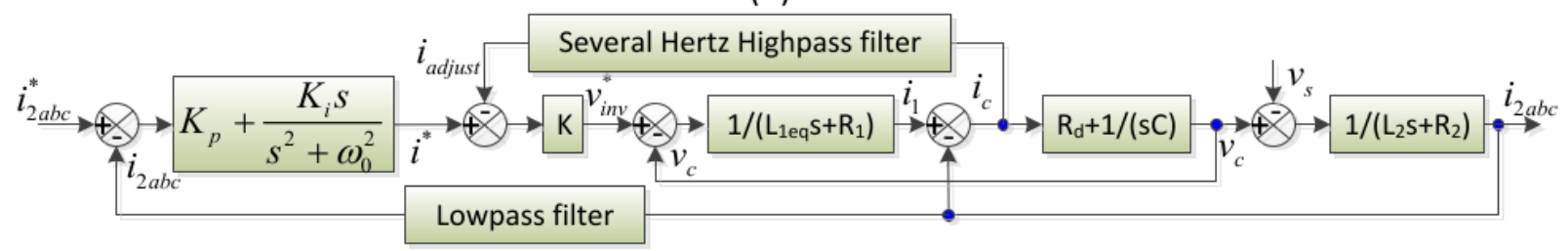

(c)

Figure 4 Inverter control flow.

The control parameters $K_{p}, K_{i}$, and $K$ for the inverter are obtained using Eqs. (4)-(10) derived from the control flow illustrated in Figure $4 \mathrm{a}$ and $4 \mathrm{~b}$.

$$
\frac{i_{2 a b c}}{\Delta i_{2 a b c}^{*}}=\frac{K \cdot\left(s C_{1}\right) \cdot\left(s R_{d} C_{1}+1\right) \cdot \text { BPFilterDen }}{D E N 1+D E N 2+D E N 3+D E N 4+D E N 5},
$$

where

$$
\begin{gathered}
\text { DEN1 }=\left[s C_{1} \cdot\left(s L_{2}+R_{2}\right)+\left(s R_{d} C_{1}+1\right)\right] \cdot\left(s L_{1}+R_{1}\right) \cdot \text { BPFilterDen } \cdot s C_{1}, \\
\text { DEN2 }=K \cdot \text { BPFilterNum } \cdot s C_{1} \cdot\left[s C_{1} \cdot\left(s L_{2}+R_{2}\right)+\left(s R_{d} C_{1}+1\right)\right],
\end{gathered}
$$




$$
\begin{gathered}
\text { DEN3 }=\left(s R_{d} C_{1}+1\right) \cdot \text { BPFilterDen } \cdot\left[s C_{1} \cdot\left(s L_{2}+R_{2}\right)+\left(s R_{d} C_{1}+1\right)\right], \\
\text { DEN4 }=K \cdot \text { BPFilterNum } \cdot s C_{1} \cdot\left(s R_{d} C_{1}+1\right), \\
\text { DEN5 }=\text { BPFilterDen } \cdot\left(s R_{d} C_{1}+1\right)^{2} .
\end{gathered}
$$

For notch-filter-based band-pass filter:

$$
\begin{gathered}
\text { BPFilterNum }=k_{d} \cdot b_{n} s, \\
\text { BPFilterDen }=s^{2}+b_{n} s+\omega_{m}^{2} .
\end{gathered}
$$

For low-pass filter + high-pass filter:

$$
\begin{gathered}
\text { BPFilterNum }=k_{d} \cdot s \cdot \text { LPCoeff } 2, \\
\text { BPFilterDen }=(s+\text { HPCoeff }) \cdot\left(s^{2}+L P C o e f f 1 \cdot s+L P C o e f f 2\right) .
\end{gathered}
$$

The capacitor current active damping is considered by applying $k_{d}$ as shown in Eqs. (10) and (12). A MATLAB command can be used for the closed-loop transfer function:

$$
\frac{i_{2 a b c}}{i_{2 a b c}^{*}}=\text { feedback }\left(\frac{i_{2 a b c}}{\Delta i_{2 a b c}^{*}}, \text { Low Pass Filter }\right) \text {, }
$$

where the low-pass filter is a second-order Butterworth filter with a cutoff switching frequency of 5 $\mathrm{kHz}$. In practice, such a filter can be replaced by choosing a small $K_{p}$ to reduce the influence of the DC component in $i_{2}$, and the PR controller can remove the high-frequency noise present in $i_{2}$.

Instead of using a band-pass filter, a high-pass filter can be used to process $i_{c}$ as shown in Figure $4 c[10,11]$.

Instead of using controllers shown in Figure 4, other controllers where the grid-side current is controlled at the outer loop as described in $[10,11]$ can be employed.

The parameters for an inverter working at $1.2 \mathrm{kV}_{\mathrm{ac}}$ are listed in Table 2.

Table 2 Parameters for the inverter.

\begin{tabular}{ll}
\hline Parameters & Values \\
\hline $\mathrm{L}_{\text {inv }}$ & $8.25 \mathrm{mH}$ \\
$\mathrm{C}_{\text {inv }}$ & $30 \mu \mathrm{F}$ \\
$\mathrm{L}_{\text {2inv }}$ & $0.5 \mathrm{mH}$ \\
fswitching & $5 \mathrm{kHz}$ \\
$\mathrm{Kp}$ & 0.15 \\
$\mathrm{Ki}$ & 10.5 \\
$\mathrm{~K}$ & 22.5 \\
fsampling & $150 \mathrm{kHz}$ \\
$\mathrm{K}_{\mathrm{v} 0}$ & 2000 \\
$\mathrm{~K}_{\mathrm{v}}$ & 1.00 \\
\hline
\end{tabular}


Table 3 shows the coefficients for the proposed filters. When the notch-filter-based band-pass filter is used, the system works even after a slight increase in $\omega_{\mathrm{m}}$.

Table 3 Parameters for the band-pass filter shown in Figure 4.

\begin{tabular}{lll}
\hline Filter & Coeff & Values \\
\hline Notch-filter-based band-pass filter & $b_{\mathrm{n}}$ & 8000 \\
& $\omega_{\mathrm{m}}$ & $2 \pi \times 50$ \\
Low-pass + High-pass filters & HPCoeff & 50 \\
& LPCoeff1 & 8887.0 \\
& LPCoeff2 & $3.949 \mathrm{e} 07$ \\
$\mathrm{k}_{\mathrm{d}}$ & 1.0 & \\
\hline
\end{tabular}

The step response and Bode plot of the closed-loop transfer function for the notch-filter-based band-pass filter are shown in Figure 5 and Figure 6, respectively, and the step response and Bode plot of the closed-loop transfer function for the case with low-pass filter plus high-pass filter are shown in Figure 7 and Figure 8, respectively, with $k_{d}=1$. These two sets of results reveal that the two approaches have a similar effect in terms of step-response convergence and stability.

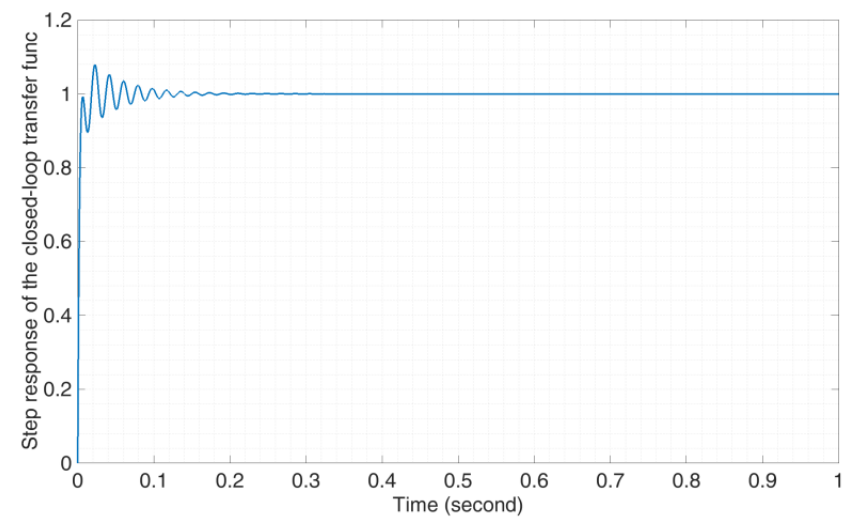

Figure 5 Step response of the closed-loop transfer function for the notch-filter based band-pass filter approach.
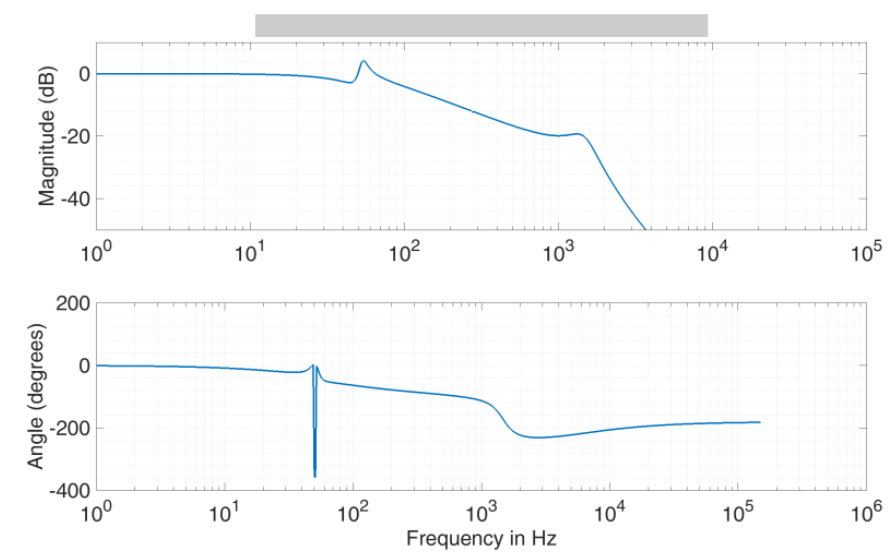

Figure 6 Bode plot of the closed-loop transfer function for the notch-filter-based bandpass filter approach. 


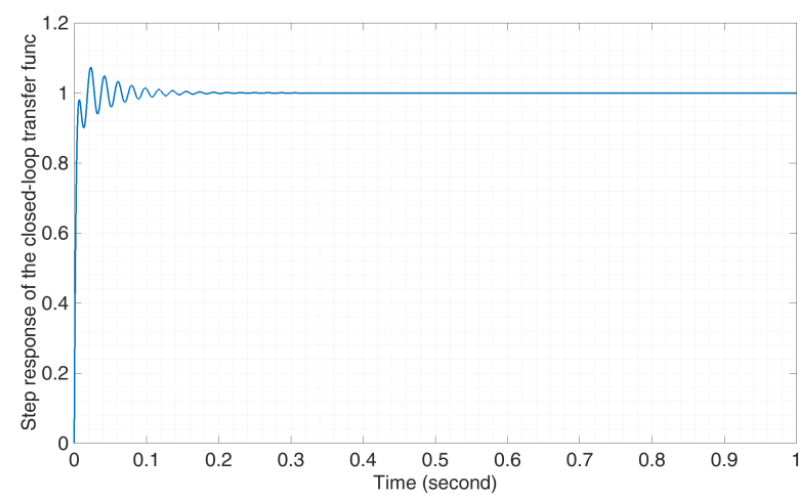

Figure 7 Step response of the closed-loop transfer function for the low-pass filter plus high-pass filter approach.
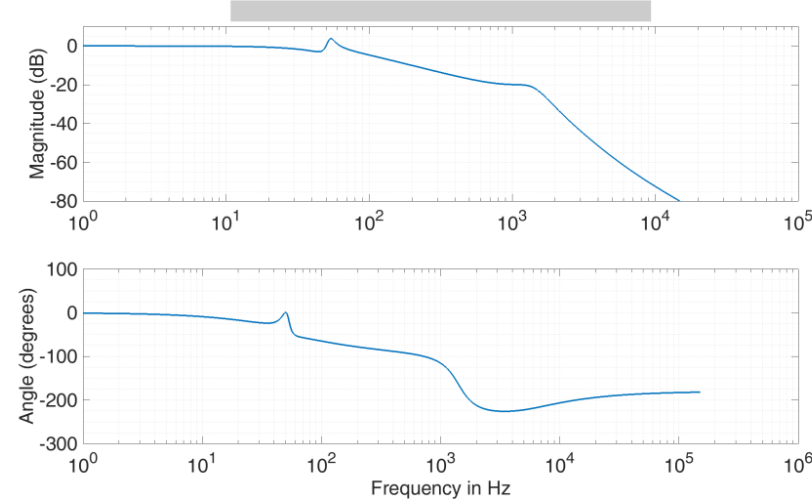

Figure 8 Bode plot of the closed-loop transfer function for the low-pass filter plus highpass filter approach.

The load information is presented in Table 4.

Table 4 Load Information.

\begin{tabular}{|c|c|c|c|c|}
\hline \multicolumn{2}{|c|}{ Load } & $P(k W)$ & $\mathrm{Q}$ (kVAr) & State \\
\hline \multirow{5}{*}{\multicolumn{2}{|c|}{1}} & 120 & 80 & Initial: Off \\
\hline & & & & On: $2.00 \mathrm{~s}$ \\
\hline & & & & Off: $2.50 \mathrm{~s}$ \\
\hline & & & & On: $4.50 \mathrm{~s}$ \\
\hline & & & & Off: $5.00 \mathrm{~s}$ \\
\hline \multirow{2}{*}{\multicolumn{2}{|c|}{2}} & 100 & 60 & Initial: Off \\
\hline & & & & On: $3.50 \mathrm{~s}$ \\
\hline 3 & & 80 & 50 & On \\
\hline 4 & & 80 & 50 & On \\
\hline \multirow[t]{2}{*}{5} & & 75 & 50 & On \\
\hline & Balanced & 150 & 80 & On \\
\hline 6 & $\begin{array}{l}\text { Unbalanced } \\
\text { (A-N) }\end{array}$ & 80 & 0 & On \\
\hline
\end{tabular}


The steps for curtailing the output power of PV panels are shown in Figure 9. As described in a previous study [12], the terminal characteristics of the PV panels can be obtained using MATLAB/Simulink modeling and a table with four-column inputs-irradiance levels, terminal currents and terminal voltages for maximum power to occur, and maximum power at each irradiance level-can be built. The polyfit command in MATLAB can then be used to obtain the coefficients for irradiance $(x)$ against maximum power $(y)$ and coefficients for maximum power $(x)$ against terminal currents $(y)$. These coefficients are used to produce reference current for controlling PV panels, as shown in Figure 9.

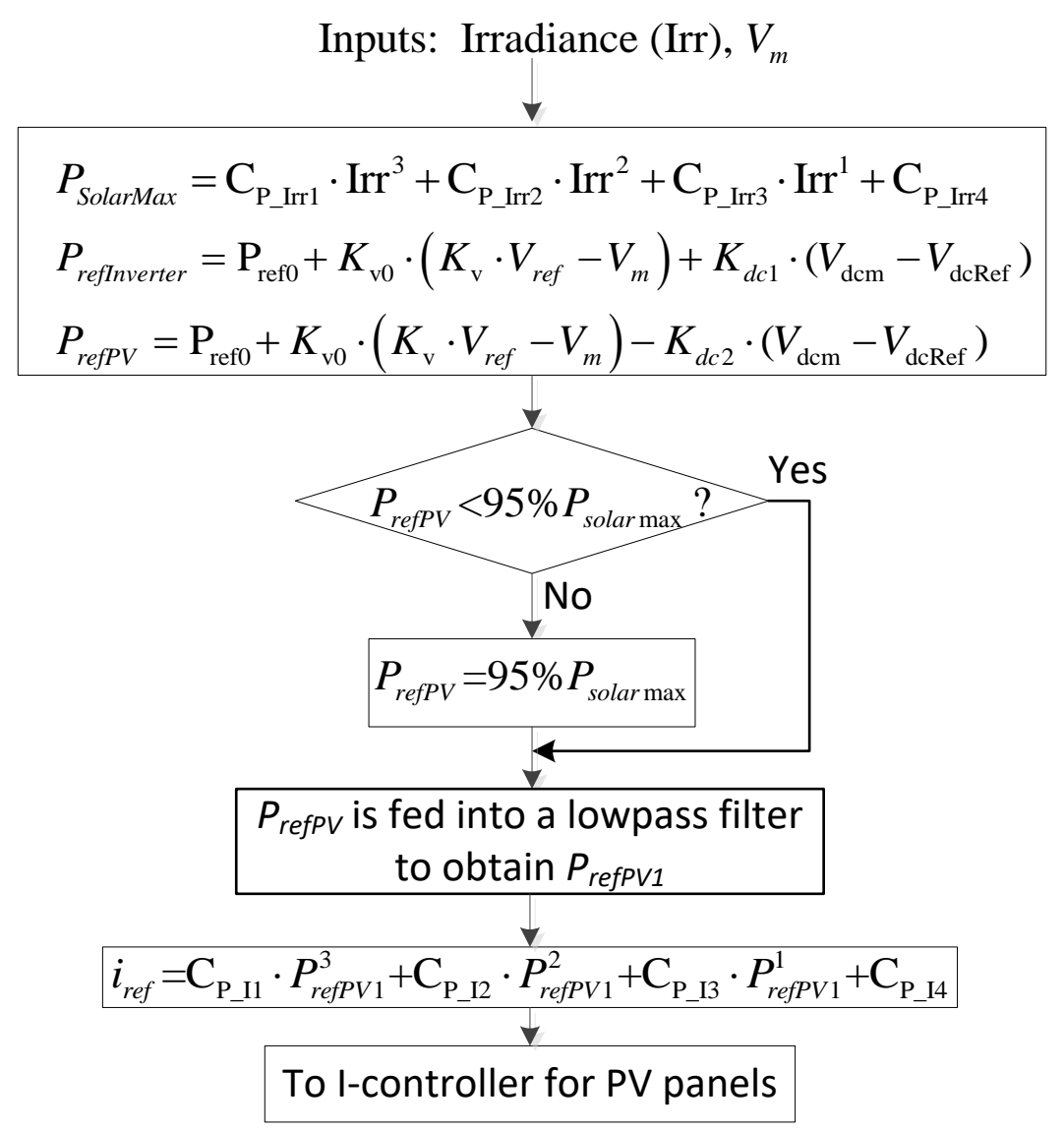

Figure 9 Flowchart showing the steps required for producing current reference for controlling PV panels.

Furthermore, the $\Delta-Y$ transformer at the load side is used to reduce the influence of unbalanced loads. Negative-sequence current compensation can also be used at the $Y$ side to further reduce the unbalance degree seen by the inverters.

\section{Results and Discussions}

\subsection{Results}

The results for filter 1 obtained using Eqs. (10) and (11) are shown in Figures 10-15. From Figure 10 , it can be seen that the voltages settle down within $\pm 5 \%$, especially after $2.5 \mathrm{~s}$, as synchronization and connection between the AC microgrid and the main grid has been accomplished. 

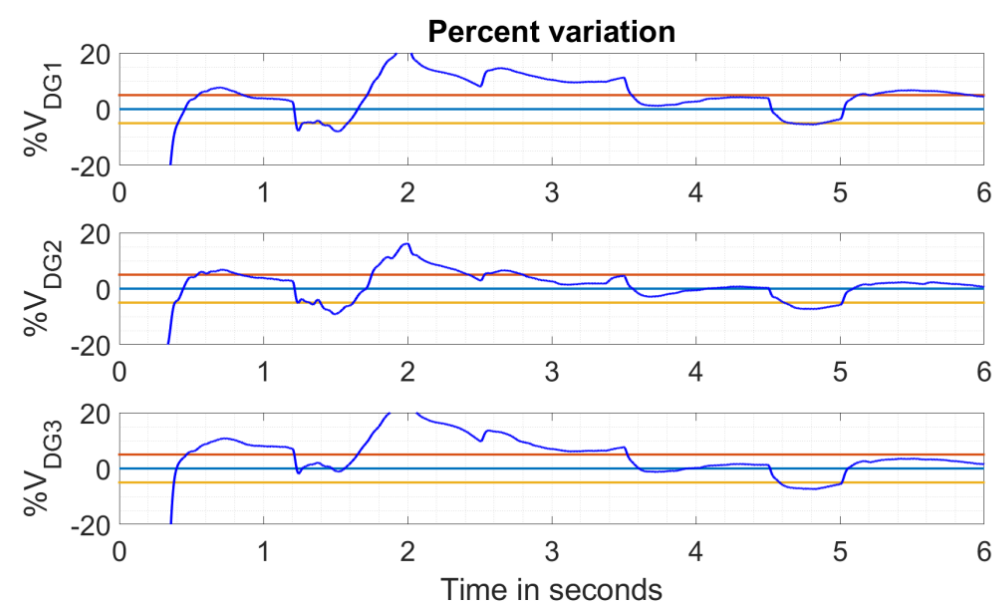

Figure 10 Variations in the voltage (in percent) at each terminal of the inverter using filter 1.

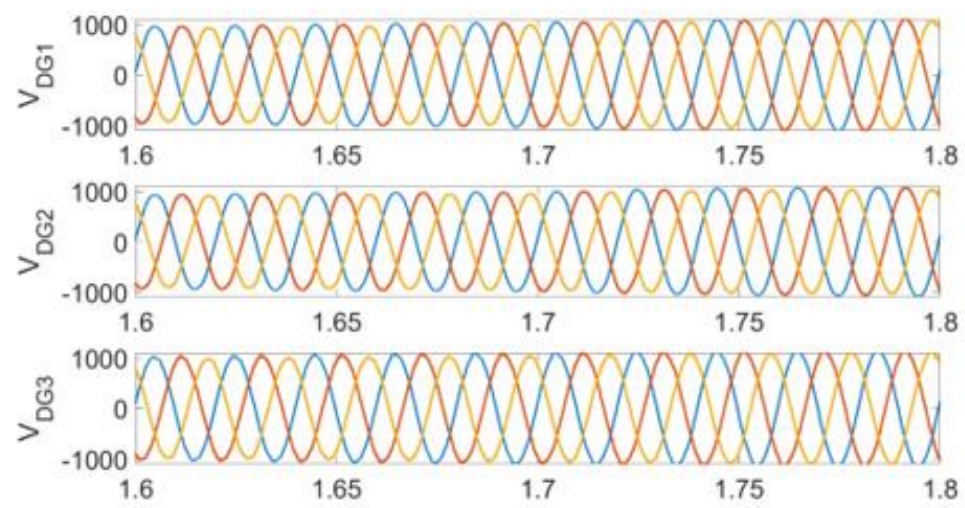

(a)
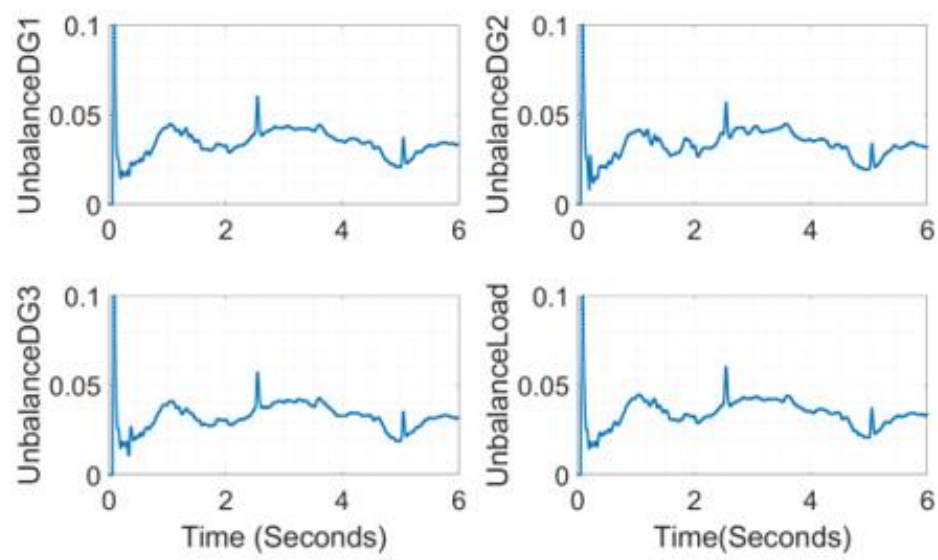

(b)

Figure 11 (a) Voltages at the inverter's terminals for a short duration when using filter 1; (b) Unbalance percentage. 

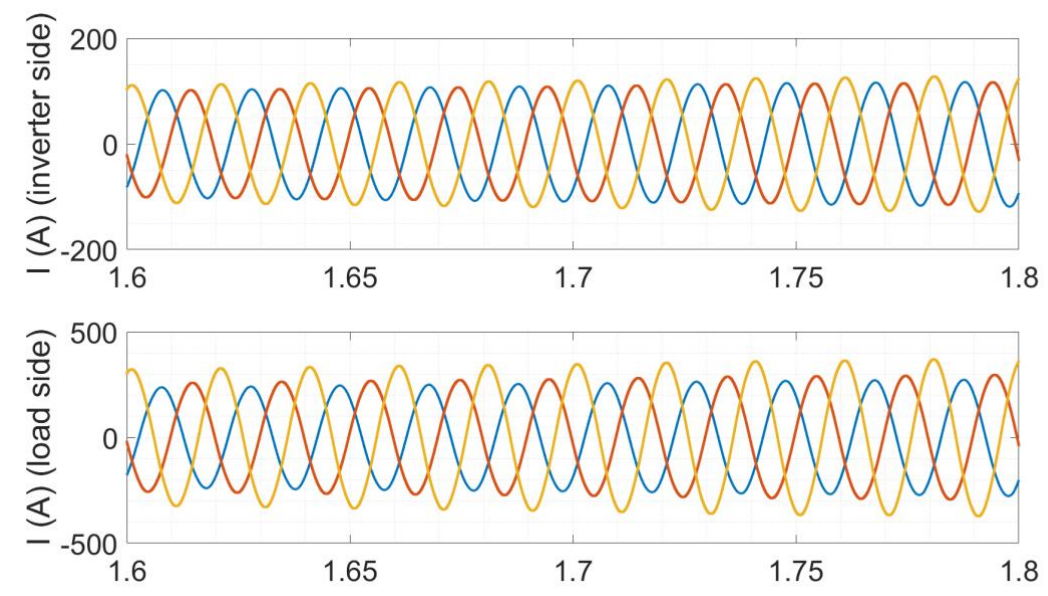

Figure 12 Currents drawn by unbalanced loads using filter 1.

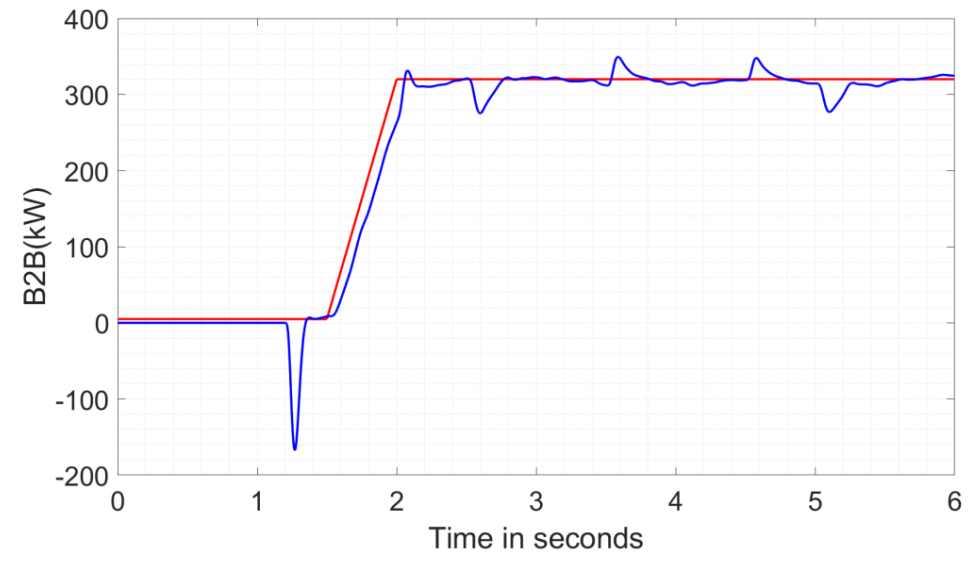

Figure 13 Planned power transfer from the large grid to the AC microgrid using filter 1.
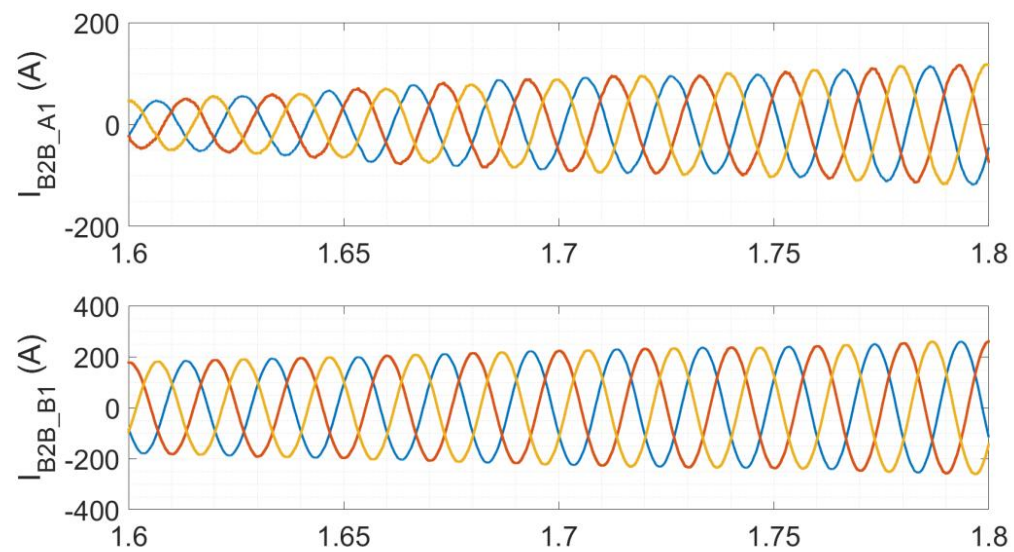

Figure 14 Currents flowing from the large grid to the AC microgrid using filter 1. 

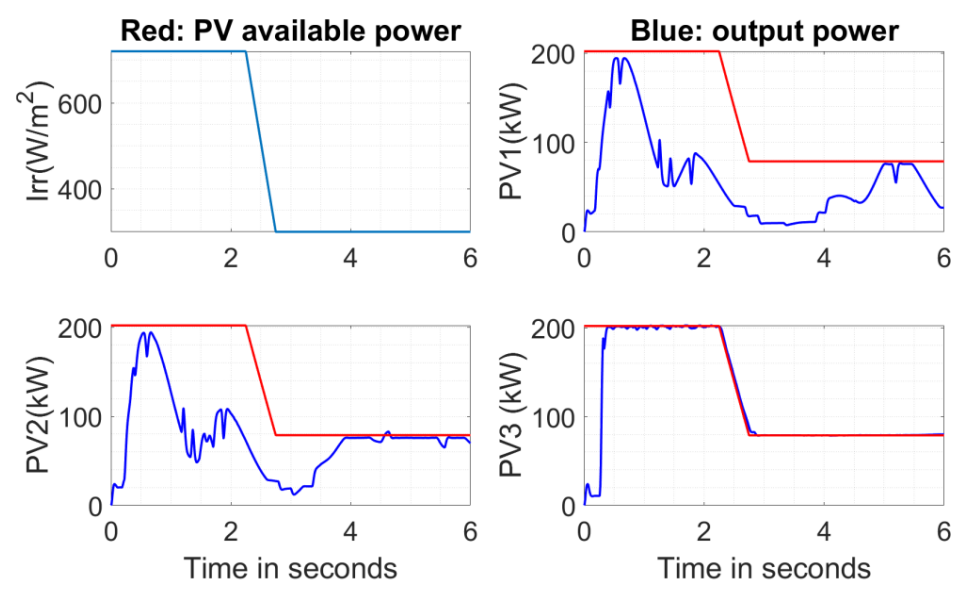

Figure 15 Variations in the irradiance and PV panel outputs using filter 1.

Due to the unbalanced loads, the voltages at the inverter's terminals become slightly unbalanced. The percent unbalance is given as follows:

$$
\begin{aligned}
& \text { Unbalance }=\sqrt{\frac{1-\sqrt{3-6 \beta}}{1+\sqrt{3-6 \beta}}}, \\
& \text { where } \beta=\frac{V_{a b}^{4}+V_{b c}^{4}+V_{c a}^{4}}{\left(V_{a b}^{2}+V_{b c}^{2}+V_{c a}^{2}\right)^{2}} .
\end{aligned}
$$

Figure 11a shows the inverter's terminal voltages for a short duration when using filter 1, whereas Figure $11 \mathrm{~b}$ shows the unbalance (in percent) for the inverter's terminal voltages and the unbalanced load's terminal voltage. These figures show that due to the $80-\mathrm{kW}$ single-phase load connected at the load side, there is an unbalance of around $4.0 \%$.

Unbalanced current from Load 6 is shown in Figure 12, where the bottom panel one depicts the current seen at the unbalanced load and the top panel depicts the current seen from the inverter side. These figures show that the $\Delta-Y$ transformer blocks zero-sequence current and makes the current at the high-voltage side less unbalanced. With reduced unbalance of the current at the $\Delta$ side or high-voltage side of the transformer, the voltage drop along the transmission lines becomes less unbalanced. Therefore, the voltage seen by the inverter at the generation side also becomes less unbalanced.

Figure 13 shows the power transfer from the large grid to the AC microgrid, where the red curve depicts the planned power transfer on the large-grid side, and the blue curve depicts the power transferred through the inverter on the AC-microgrid side. Figure 13 shows that the power transferred can follow the planned power transfer closely; the small difference is due to losses in the AC/DC/AC converter.

The currents flowing for a short duration through the two converters in the AC/DC/AC converter are illustrated in Figure 14.

Figure 15 shows the variations in the irradiance, and reference power and output power from each PV panel, PV1, PV2, and PV3. The PV1-powered inverter serves as the grid-forming generator, while the PV2-powered inverter serves as the grid--supporting generator; both adopt the approach of real power generation given by Eq. (1) and follow the steps described in Figure 9. Therefore, their 
output power varies with load changes and the power transferred by the AC/DC/AC converter. The PV3-powered inverter serves as the grid-feeding generator, with the PV panel controlled using the maximum power point tracking mode. From the right-bottom panel in Figure 15, it can be seen that the output power of the PV panel follows its reference quite closely.

The results for filter 2 obtained using Eqs. (12) and (13) are shown in Figures 16-21. Similar to those for filter 1 , the voltage level in the system is maintained well, and the inverters can cope with unbalanced loads.
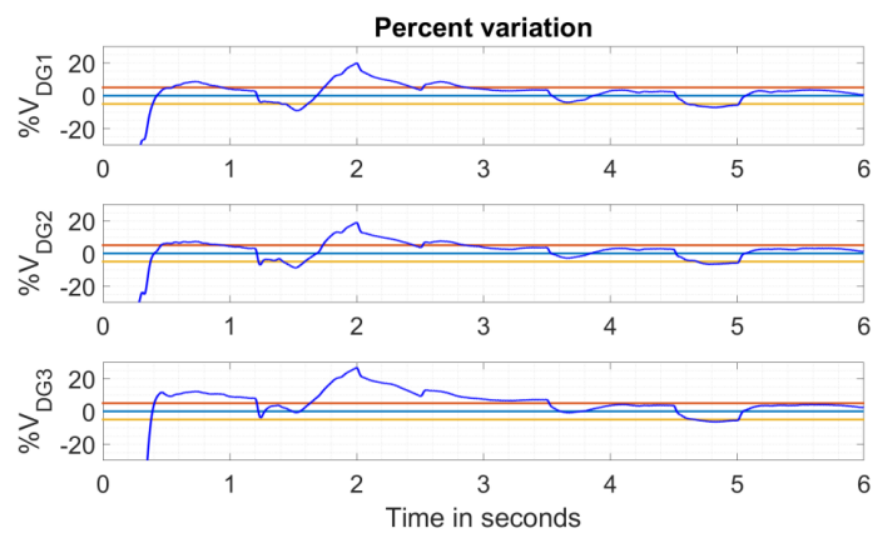

Figure 16 Variations in the voltage (in pecent) at each terminal of the inverter using filter 2.

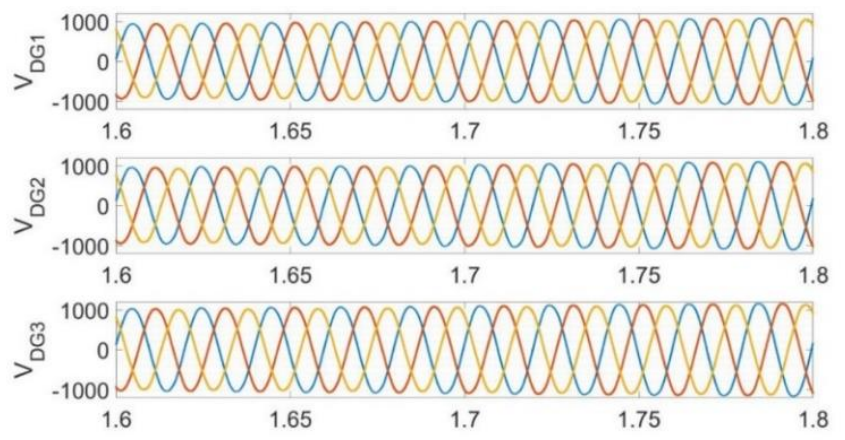

(a)
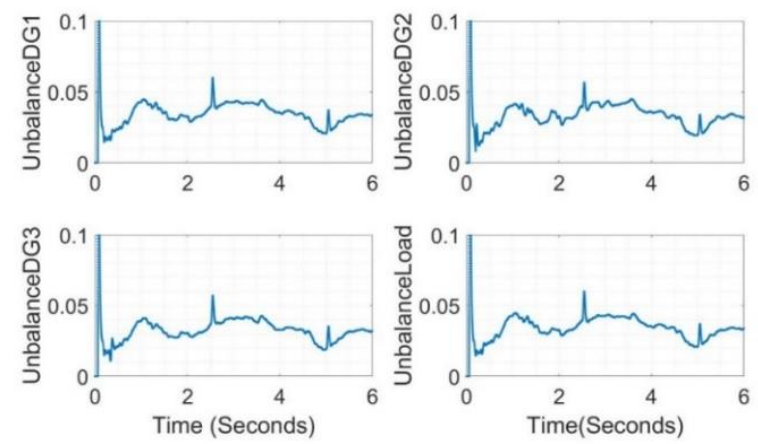

(b)

Figure 17 (a) Voltages at the inverter's terminals for a short duration using filter 2; (b) Unbalance percentage. 
JEPT 2022; 4(1), doi:10.21926/jept.2201008
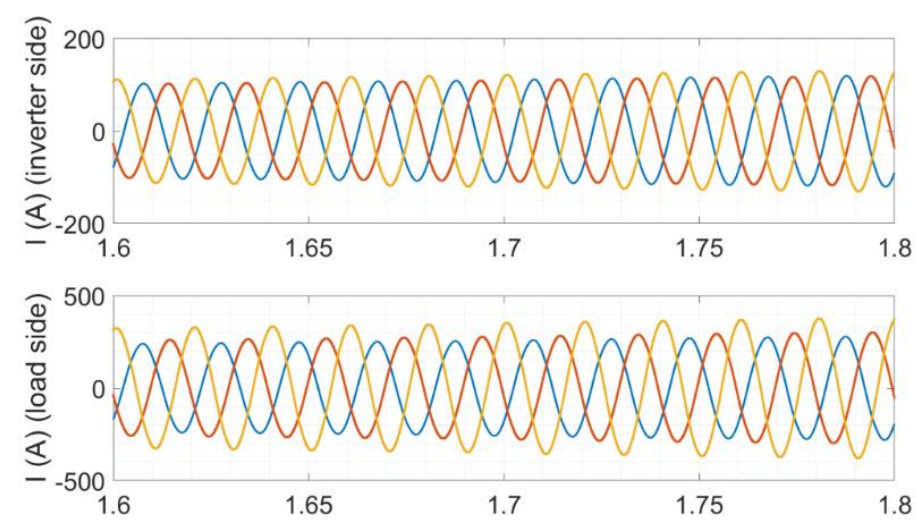

Figure 18 Currents drawn by unbalanced loads using filter 2 .

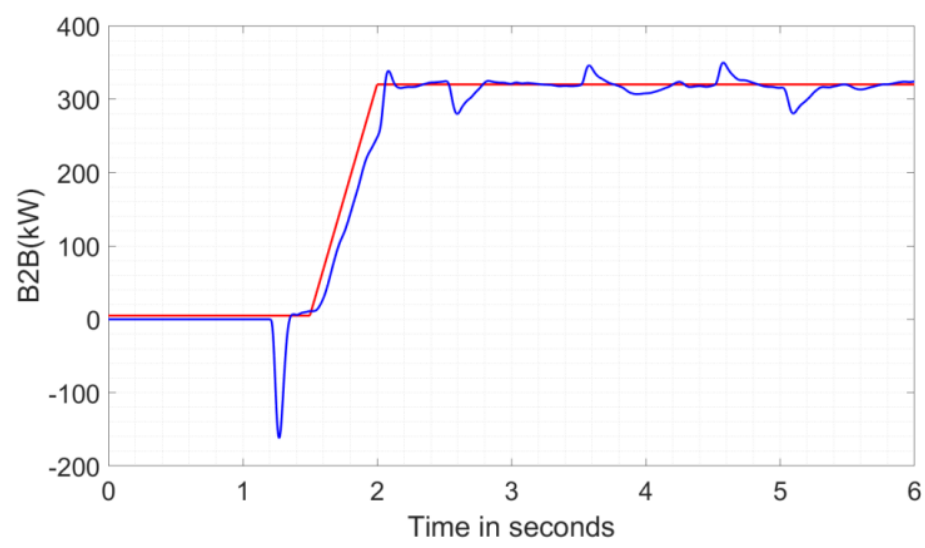

Figure 19 Planned power transfer from the large grid to the AC microgrid using filter 2.
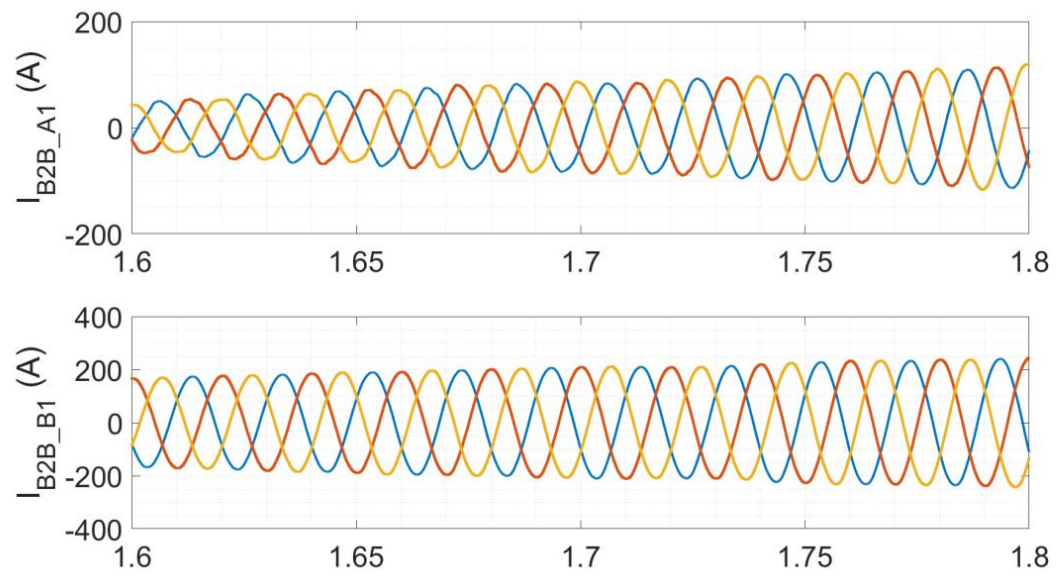

Figure 20 Currents flowing from the large grid to the AC microgrid using filter 2. 

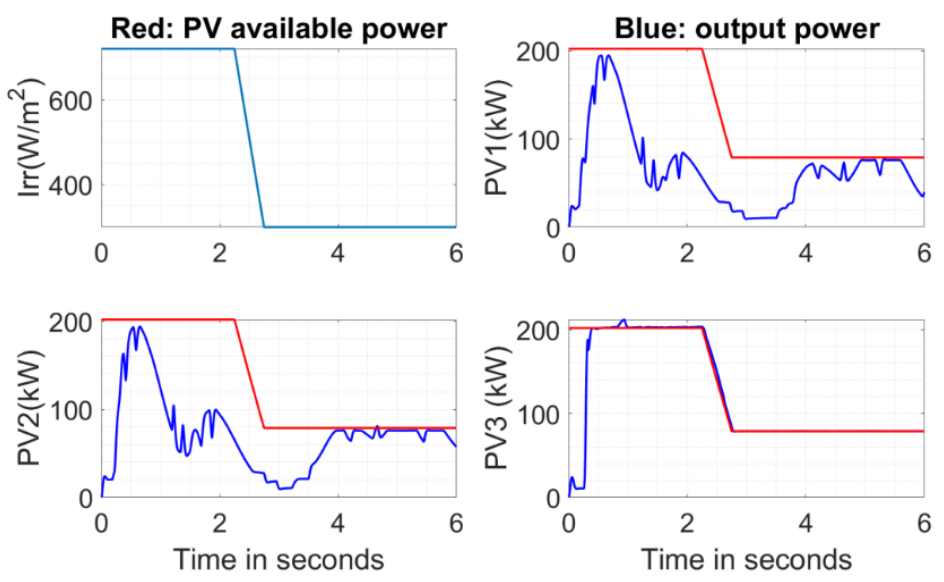

Figure 21 Variations in the irradiance and PV panel outputs using filter 2.

For both cases, the system can work with only PV panels in islanded mode before $1.20 \mathrm{~s}$ after startup. At $1.20 \mathrm{~s}$, the $A C / D C / A C$ converter is synchronized with the $A C$ microgrid and power transferring from the large grid to the AC microgrid happens at $1.5 \mathrm{~s}$. With more power transferred, the outputs from each of the PV1 and PV2 are pushed to be smaller. By doing so, the voltage profiles at each bus in the AC microgrid are maintained relatively good, as can be seen in Figures 10 and 16 . By adjusting $K_{v}$, the voltage profiles can be tuned to be better.

From Figures 15 and 21, it can be seen that filter 1 and filter 2 exhibit similar performance in terms of curtailment of the PV panels' output.

In the above study, the PV panel control for the grid-forming generator DG1 and the gridsupporting generator DG2 is in curtailment mode, as described in Figure 9. Thus, it is feasible to use PV panels without energy storage systems to run AC microgrids smoothly on sunny days. Before the grid connection is performed at $1.2 \mathrm{~s}$, the AC microgrid operates in the autonomous mode. Thus, such an operation can be off-grid on sunny days and grid-connected mode in other weather conditions. This approach has practical applications as frequent charging and discharging of battery storage accelerates its aging. Autonomous or standalone operation is suitable only for sunny days. By doubling the number of solar panels, the system can operate in autonomous or standalone mode with sufficient power output even under slightly cloudy weather conditions.

In the operation described above, only PV1 and PV2 used for DG1 and DG2, respectively, adopt the curtailment operation. Certainly, all the PV panels can adopt curtailment operation.

For practical operation, the PV panel and its DC/DC converter in the grid-forming generator can be replaced by a DC source such as a battery set or a supercapacitor unit. In a subsequent numerical experiment, PV1 and its DC/DC converter are replaced by a DC source. The PV panels' control for DG2 is still in curtailment mode, and PV panels' control for DG3 is still in maximum power point tracking mode. Some of the results for this operation are displayed in Figures 22 and 23 . These results show that such an operation is equally good. 

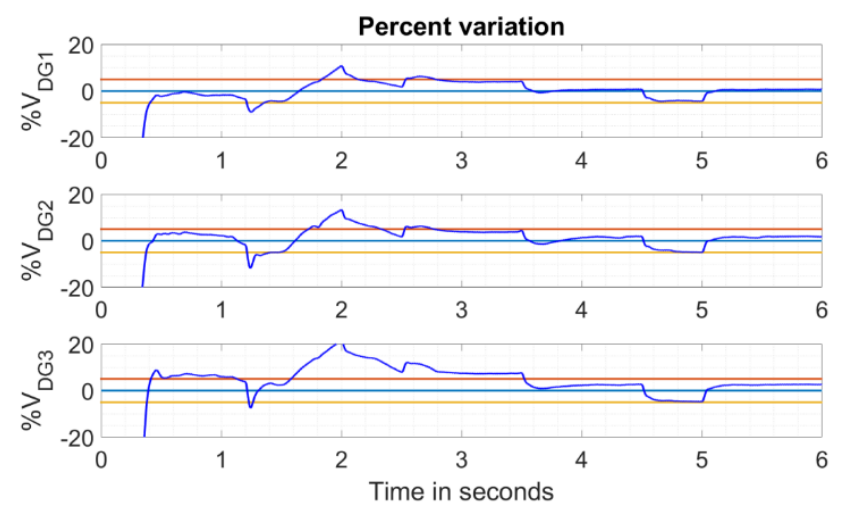

Figure 22 Variations in the voltage (in percent) at each terminal of the inverter using filter 2.
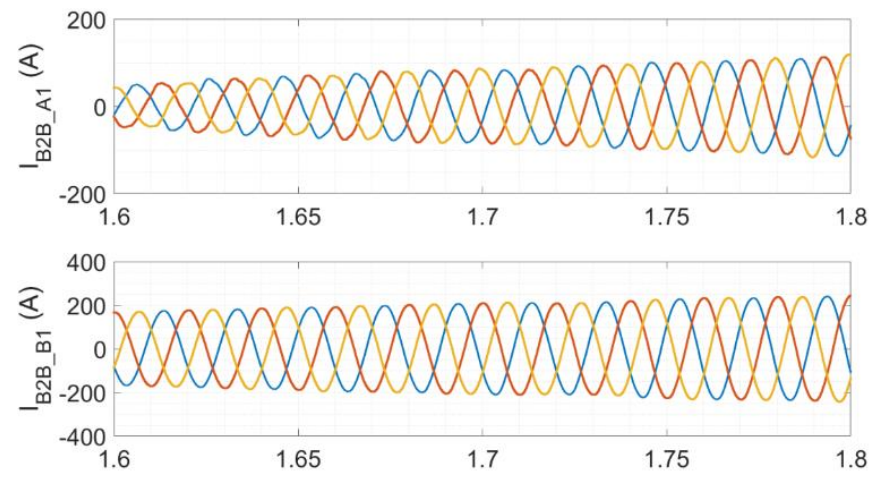

Figure 23 Currents flowing from the main grid to the microgrid using filter 2 .

In this study, MPPT proposed in previous studies [6-8] was adopted for achieving maximum power extraction from PV panels. Other MPPT methods [13-15] can also be used.

\subsection{Discussions}

Although we advocate minimizing the usage of energy storage close to the location of end-users, large energy storage systems can be built in remote locations where large solar farms and/or wind farms are located. The storage system can be gravitational potential energy storage plus supercapacitor buffer [16]; alternatively, moderate battery storage capacity with a well-monitored retrieval system can be employed. These large energy storage systems can provide necessary support through a high-voltage interface, for example, at $11 \mathrm{kV}$, as shown in Figure 1. Nevertheless, they can be operated at low voltages by using multiple SSTs in parallel for interfacing with the AC grid through a step-up transformer and transmission lines. The step-up transformer increases the step voltage to $11 \mathrm{kV}$. On the transmission line side close to the storage farm, one of the SSTs works as the grid-forming generator with a constant-frequency reference voltage. One or two SSTs work in tandem with the grid-forming SST to cope with transient reactive power demands. Other SSTs work as grid-supporting generators. These SSTs can be AC $(50 \mathrm{~Hz})$-DC-AC (medium-frequency)medium-frequency transformers with passive filter-AC (medium-frequency)-DC-AC $(50 \mathrm{~Hz})$ converters if storage units interface with an $A C$ microgrid or DC-AC (medium-frequency)-medium- 
frequency transformer with passive filter-AC (medium-frequency)-DC-AC ( $50 \mathrm{~Hz}$ ) if the storage units are interfaced with a DC microgrid. The storage is charged using another DC microgrid.

Conventional synchronous generators can be employed as a backup; they can be interfaced with the AC microgrid through multiple parallel SSTs with medium-frequency transformer isolation.

\section{Conclusions}

In this paper, we proposed a method that involves the use of PV panels without energy storage to power inverters in an AC microgrid. The proposed system is operated at a constant frequency. With sufficient daylight intensity, such a system can be operated either in standalone mode or in grid-connected mode.

When the intensity of daylight is low, power can be imported from the main grid through an $A C / D C / A C$ converter to compensate for the shortage. Numerical results revealed that with an accurate prediction of the weather and the power to be imported from the large power system, AC microgrids could be run smoothly.

Furthermore, in this paper, we proposed the construction of large energy storage farms at more convenient locations. They can be used to replace large grids to transfer power to multiple AC microgrids when necessary.

By using a three-phase transformer in $\Delta-Y$ connection with $Y$ 's neutral connected to its local grounding system, the effect of unbalanced loads on the stability of the AC microgrid can be minimized. To power more unbalanced loads, we proposed the use of negative-sequence current compensators for minimum change in the existing power system infrastructure at the user end.

By operating the microgrid at a high voltage, more power can be transferred.

\section{Author Contributions}

The author did all the research work of this study.

\section{Competing Interests}

The author has declared that no competing interests exist.

\section{References}

1. Olivares DE, Mehrizi-Sani A, Etemadi AH, Cañizares CA, Iravani R, Kazerani M, et al. Trends in microgrid control. IEEE Trans Smart Grid. 2014; 5: 1905-1919.

2. Lee CT, Chu CC, Cheng PT. A new droop control method for the autonomous operation of distributed energy resource interface converters. IEEE Trans Power Electron. 2012; 28: 19801993.

3. Mohamed YA, El-Saadany EF. Adaptive decentralized droop controller to preserve power sharing stability of paralleled inverters in distributed generation microgrids. IEEE Trans Power Electron. 2008; 23: 2806-2816.

4. Hassan MA, Abido MA. Optimal design of microgrids in autonomous and grid-connected modes using particle swarm optimization. IEEE Trans Power Electron. 2010; 26: 755-769. 
5. Babu TS, Vasudevan KR, Ramachandaramurthy VK, Sani SB, Chemud S, Lajim RM. A comprehensive review of hybrid energy storage systems: Converter topologies, control strategies and future prospects. IEEE Access. 2020; 8: 148702-148721.

6. Zhang D. Constant frequency operated autonomous AC microgrid with different MPPT methods applied to PV panels and with converting circuit for powering unbalanced loads. Hangzhou: Asia Conference on Power and Electrical Engineering; 2022.

7. Zhang D, Fletcher J. Design and operation of an islanded microgrid at constant frequency book chapter in microgrid. Rijeka: InTech; 2017.

8. Zhang D. Issues on load shedding in a microgrid operated at constant frequency. Proceedings of the 2017 20th International Conference on Electrical Machines and Systems; 2017 August 11th; Sydney, NSW, Australia. Piscataway: Institute of Electrical and Electronics Engineers.

9. Zhang D. Issues on autonomous ac microgrid operated at constant frequency. Proceedings of the 2021 31st Australasian Universities Power Engineering Conference; 2021 September 26th; Perth, Australia. Piscataway: Institute of Electrical and Electronics Engineers.

10. Liu Q, Caldognetto T, Buso S. Review and comparison of grid-tied inverter controllers in microgrids. IEEE Trans Power Electron. 2019; 35: 7624-7639.

11. Han Y, Li Z, Guerrero JM. Dynamic evaluation of LCL-type grid-connected inverters with different current feedback control schemes. Proceedings of the 2015 9th International Conference on Power Electronics and ECCE Asia; 2015 June 1st; Seoul, South Korea. Piscataway: Institute of Electrical and Electronics Engineers.

12. Zhang D, Tseng K. A universal controller for grid-tied DC/AC converters for harnessing PV panel based solar energy and PMSG based wind energy. Proceedings of the 2015 IEEE 11th International Conference on Power Electronics and Drive Systems; 2015 June 9th; Sydney, NSW, Australia. Piscataway: Institute of Electrical and Electronics Engineers.

13. Podder AK, Roy NK, Pota HR. MPPT methods for solar PV systems: A critical review based on tracking nature. IET Renew Power Gener. 2019; 13: 1615-1632.

14. TOZLU ÖF, ÇALIK H. A review and classification of most used MPPT algorithms for photovoltaic systems. Hittite J Sci Eng. 2021; 8: 207-220.

15. El-Khozondar HJ, El-Khozondar RJ, Matter K, Suntio T. A review study of photovoltaic array maximum power tracking algorithms. Renewables. 2016; 3. Doi: 10.1186/s40807-016-0022-8.

16. Zhang D. High-speed heavy mass energy storage system with multiple identical units. J Multidiscip Eng Sci Technol. 2020; 7: 11892-11898. 


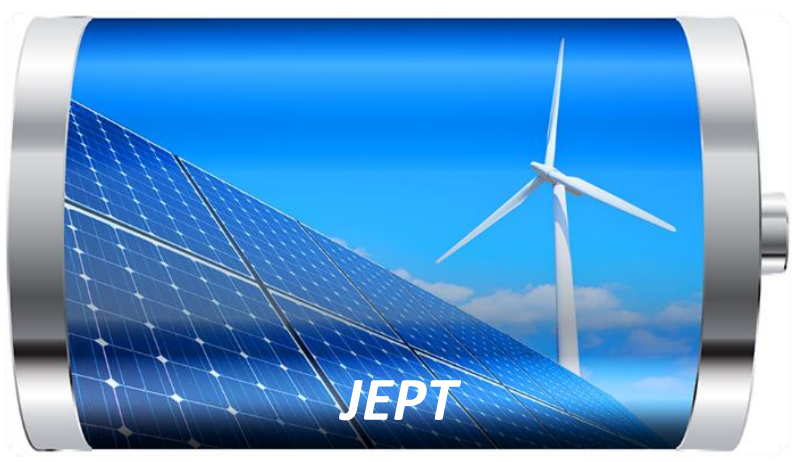

Enjoy JEPT by:

1. Submitting a manuscript

2. Joining in volunteer reviewer bank

3. Joining Editorial Board

4. Guest editing a special issue

For more details, please visit:

http://www.lidsen.com/journal/jept 